ARTICLE

https://doi.org/10.1038/s41467-019-10098-z

\title{
Simple non-fused electron acceptors for efficient and stable organic solar cells
}

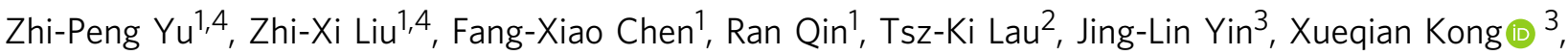 \\ Xinhui Lu (i) ${ }^{2}$, Minmin Shi ${ }^{1}$, Chang-Zhi Li ${ }^{1} \&$ Hongzheng Chen ${ }^{1}$
}

The flexibility in structural design of organic semiconductors endows organic solar cells (OSCs) not only great function-tunabilities, but also high potential toward practical application. In this work, simple non-fused-ring electron acceptors are developed through twostep synthesis from single aromatic units for constructing efficient OSCs. With the assistance of non-covalent interactions, these rotatable non-fused acceptors (in solution) allow transiting into planar and stackable conformation in condensed solid, promoting acceptors not only feasible solution-processability, but also excellent film characteristics. As results, decent power conversion efficiencies of $10.27 \%$ and $13.97 \%$ can be achieved in single and tandem OSCs consisting of simple solution-cast blends, in which the fully unfused acceptors exhibit exceptionally low synthetic complexity index. In addition, the unfused acceptor and its based OSCs exhibit promising stabilities under continuous illumination. Overall, this work reveals valuable insights on the structural design of simple and effective electron acceptors with great practical perspectives.

\footnotetext{
${ }^{1}$ State Key Laboratory of Silicon Materials, MOE Key Laboratory of Macromolecular Synthesis and Functionalization, Department of Polymer Science and Engineering, Zhejiang University, 310027 Hangzhou, P. R. China. ${ }^{2}$ Department of Physics, The Chinese University of Hong Kong, New Territories, 999077 Hong Kong, P. R. China. ${ }^{3}$ Department of Chemistry, Zhejiang University, 310027 Hangzhou, P. R. China. ${ }^{4}$ These authors contributed equally: Zhi-Peng Yu, Zhi-Xi Liu. Correspondence and requests for materials should be addressed to C.-Z.L. (email: czli@zju.edu.cn)
} 
A s one of the promising clean energy solutions, the organic solar cell (OSC) possesses unique features distinct from inorganic ones, such as lightweight and flexibility, as well as the great function tunabilities rooting from the structural versatilities of organic semiconductors ${ }^{1,2}$. During the past few decades, OSC has experienced continuous advancement with the fast evolution of electroactive molecules. Once, OSCs commonly employed the bulk heterojunction (BHJ) of p-type polymers and/ or molecules with n-type fullerene electron acceptors ${ }^{3-6}$. Recent breakthrough of OSC performance was made possible ${ }^{1,2,7-9}$, with the dawn of fused-ring electron acceptor (FREA) at 201510,11, wherein FREA typically adapts acceptor-donor-acceptor (A-D-A) molecular architecture with a fused ladder-type core $(D)^{2}$. Fused ladder structures refer to those chemical-bondsreinforced multiple membered-ring structures (usually more than five rings), which allow potentially reducing the reorganization energy of molecules ${ }^{12,13}$. The recent development of highly efficient FREAs, all consisting of fused ladder cores, such as

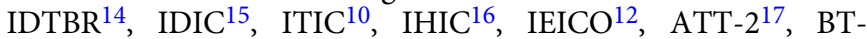
$\mathrm{CIC}^{18,19}$, DTPC-IC ${ }^{20}$, COi8DFIC ${ }^{21}$, etc. ${ }^{2,8,22}$, enables BHJ layers to effectively utilize solar photons from visible to near-infrared range (NIR). Power conversion efficiencies (PCEs) of the OSCs with FREAs have nowadays reached over $14 \%{ }^{8}$ for single junction and $17 \%^{23}$ for tandem OSCs, respectively. Although exciting progresses have been made, the derivation of FREAs has surprisingly expanded into rather complex structures, ranging from five to seven, nine, and eleven- (or more) membered rings.

While the level of OSC performance is revalent to the practical application, significant challenges, such as the accessibility of effective materials and devices, should be put under consideration for research activities, which is closely related to the scalability and costs of OSCs ${ }^{14,24,25}$. So far, the access of multi-ring-fused ladder structure inevitably involves synthetic complexities. A typical example is the indacenodithiophene (IDT) unit, the simplest fused-ring electron-donating core for FREAs reported so far, which is typically synthesized over 6 (or 4) steps, depending on either aliphatic (or aromatic) side chains attached on the bridge $s p^{3}$-carbon up-pointing and down-pointing fashion. The synthetic complexities of the molecule are inversely proportional to the industrial figure of merit for $\mathrm{OSCs}^{24,26}$, since multi-step synthesis and purification rapidly increase the material costs. These issues would potentially undermine the practical perspectives of OSCs. Therefore, despite a large family of electron acceptors developed, there is still a strong need to explore the new structural design of simple and effective molecules, for accessing efficient and low-cost OSCs.

Bearing this in mind, electron acceptors with partially ${ }^{17,27-29}$ or fully unfused backbones ${ }^{30}$, have recently been explored, wherein the noncovalent intramolecular interactions ${ }^{29,31-33}$ are employed to mediate the planarity of these molecular structures. If performance insufficiency is overcome, efficient OSCs with easy accessibility of key active components could be expected. Therefore, it is highly interesting to explore the design strategy and structure-property relation of non-fused-ring acceptors (NFRAs), particularly to embed excellent optoelectronic and stacking properties for the unfused (and potentially rotatable) molecules, which allows improving their $\mathrm{BHJ}$ characteristics and photovoltaic performance.

Herein, three exceptionally simple electron acceptors, PTICH, PTIC, and PTICO NFRAs, consisting of unfused 2,2'-(2,5-dialkyloxy-1,4-phenylene) dithiophene (PT) core and fluorinated 1,1-dicyanomethylene-3-indanone (DFIC) terminals, are developed through two-step synthesis without column purification from single aromatic units for constructing efficient OSCs. Through theoretical and experimental investigations, we reveal that NFRAs with rotatable conformation in solution could be restrained into planar and stackable conformation in condensed solid. The reasons are due to the presence of intramolecular $\mathrm{O}-\mathrm{H}$ interaction among the PT core and steric hindrance between substituted thiophene and terminal DFIC (3-position substituent on thiophene: hexyl of PTIC and hexyloxy of PTICO, rather than $\mathrm{H}$ of PTICH), wherein the former reinforces backbone planarity, and the latter prevents the structural isomerization of the terminal double bond on NFRAs. Meanwhile, as evidenced from solid-state nuclear magnetic resonance (SSNMR) and grazingincidence wide-angle X-ray scattering (GIWAXS), dense end-toend stacking of NFRAs is observed with closer $\pi-\pi$ distance than that of FREA, ID4F. These promote NFRAs with the improved optoelectronic properties in solids.

Among OSCs containing BHJs made of NFRAs and PBDB-TF polymer, decent PCEs of $10.27 \%$ and $13.97 \%$ can be achieved for the single and tandem OSCs consisting of PTIC-based BHJs, in which PTIC exhibits exceptionally low synthetic complexity index. In addition, NFRA itself, i.e., PTIC and NFRA-based OSCs exhibit promising stabilities under continuous 1-sun-equivalent illumination. Overall, we demonstrate that exceptionally simple acceptors with desirable optoelectronic properties in solids can lead to efficient and stable OSCs. This study reveals valuable insights on the structural design of simple and effective electron acceptors with great practical perspectives.

\section{Results}

Synthesis of NFRAs and molecular monformation. The fully non-fused acceptors, PTICH, PTIC, and PTICO, are designed and achieved with a two-step synthetic route (Fig. 1), which are generally more convenient than the synthesis of FREAs (Supplementary Figs. 1 and 2). The chemical structures of the studied NFRAs and polymer donors, along with a fused-ID4F acceptor are shown in Supplementary Figs. 3 and4. PT-CHOs were first constructed by Palladium (II)-catalyzed $\mathrm{C}-\mathrm{H}$ activation coupling reactions between 1,4-dibromo-2,5-bis((2-hexyldecyl)oxy) benzene and thiophene-2-carbaldehydes, featured with atomic economy. Target products were finally accessed from Knoevenagel condensation of PT-CHOs and DFIC. It is worthy to note that NFRAs were purified by recrystallization without the need of silica gel column, featured with the simple and scalable work-up processes. The final products were characterized by ${ }^{1} \mathrm{H}-\mathrm{NMR}$, ${ }^{13} \mathrm{C}-\mathrm{NMR}$, and mass spectra. The synthetic complex (SC) index of these NFRAs was also calculated according to reported methodologies ${ }^{24,34}$, wherein the SC index of PTIC is around 55\%, much smaller than that of ID4F (97\%) and other compared FREAs (generally beyond 80\%) (Supplementary Table 1). Essentially, the smaller SCs for NFRAs were attributed from intrinsically short synthetic routes and ease of purification.

In order to understand the molecular conformation of the above NFRAs, we first conducted density functional theory (DFT) calculations (Fig. 2a and Supplementary Fig. 5). Within NFRAs, two potentially rotatable single $\mathrm{C}-\mathrm{C}$ bonds (center single bond between alkyloxybenzene and thiophene, noted as PT rotamer; terminal single bond between thiophene and DFIC, noted as TIC rotamer) were present. The relaxed potential surface energy scans of possible rotamers were performed (Fig. 2a). The energy-torsion angle curves ( $E-\theta$ curves) reveal that $\mathrm{PT}$ rotamer has a rotation energy barrier of $4.4 \mathrm{~kJ} \mathrm{~mol}^{-1}$, from the conformation with the lowest total energy $\left(10^{\circ} \mathrm{PT}\right.$ rotamer featured with $\mathrm{O}-\mathrm{H}$ interaction, $\mathrm{O}-\mathrm{H}$ geometry), through $90^{\circ} \mathrm{PT}$ rotamer, to the conformation with the second lowest total energy $\left(170^{\circ} \mathrm{PT}\right.$ rotamer featured with $\mathrm{O}-\mathrm{S}$ interaction between alkyloxybenzene and thiophene, $\mathrm{O}-\mathrm{S}$ geometry). Note that $\mathrm{O}-\mathrm{H}$ geometry has A slightly lower total energy than that of O-S geometry (Fig. 2a and Supplementary Fig. 5). It suggests that 



Fused-ring electron acceptor (FREA)

Non-fused-ring acceptor (NFRA)

b NFRA:



Solution (rotation)

Solid (restriction of rotation)

C General synthesis of NFRAs:
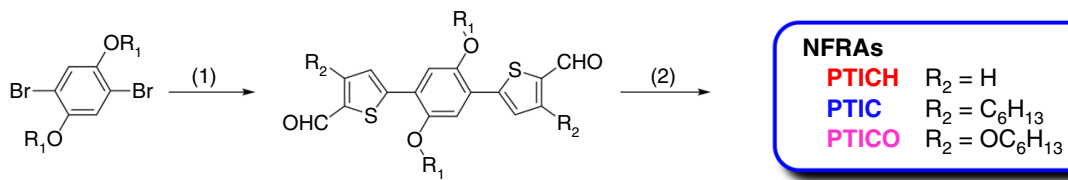

Fig. 1 Non-fused-ring acceptors (NFRAs) and their synthetic route. a The chemical structure of FREA and NFRA. b The schematic presentation of NFRA conformation rearrangement from solution to solid state. c Two-step synthetic route for NFRAs. Here $\mathrm{R}_{1}$ is 2-hexyldecyl. Reacted condition: (1) 3substituent-2-thenaldehyde, palladium-catalyzed $\mathrm{C}-\mathrm{H}$ activation coupling and (2) DFIC, Knoevenagel condensation

$\mathrm{O}-\mathrm{H}$ geometry of NFRAs is the energetically preferential conformation, which, however, can thermodynamically access $\mathrm{O}-\mathrm{S}$ geometry if rotation barrier of $\mathrm{PT}$ rotamer is being overcome. In addition, the $E-\theta$ curves of the terminal $\mathrm{C}-\mathrm{C}$ bond (TICH rotamer for PTICH, TIC rotamer for PTIC, and TICO rotamer for PTICO) were compared to indicate that substituted thiophene helps preventing the generation of a $180^{\circ}$ rotamer (one of the possible isomers for NFRAs). It is because the steric hindrance between the thiophene substituent and DFIC significantly increases energy barriers over $45 \mathrm{~kJ} \mathrm{~mol}^{-1}$ (as a comparison, TICH rotamer with unsubstituted thiophene shows only a barrier of $6.25 \mathrm{~kJ} \mathrm{~mol}^{-1}$ at torsion angle of $180^{\circ}$ ). Theoretical investigations suggest that NFRAs adapt $\mathrm{O}-\mathrm{H}$ geometry as their preferential conformation, wherein the center single bond has a relatively small rotation barrier, whereas, the terminal single bond shows less rotation tendency (Fig. 2a).

To probe the noncovalent $\mathrm{O}-\mathrm{H}$ intramolecular interaction, we further conducted temperature-dependent ${ }^{1} \mathrm{H}$-NMR for NFRAs (Fig. 2b). Interestingly, the chemical shift of thiophene proton $\left(\mathrm{H}_{\mathrm{a}}\right)$ in PTIC (in $\mathrm{d}_{4}$-dichlorobenzene) gradually moves from 7.83 to $7.70 \mathrm{ppm}$, upon increasing temperature from 30 to $120^{\circ} \mathrm{C}$. It is the characteristic for breaking hydrogen bonds between $\mathrm{H}_{\mathrm{a}}$ and oxygen atoms on alkyloxybenzene of the PT core at the elevated temperature. The hydrogen bond is also supported from the calculated $\mathrm{H}_{\mathrm{a}}-\mathrm{O}$ distance that is shorter than the sum of Van der Waals radius for $\mathrm{H}$ and $\mathrm{O}$ atoms ${ }^{35}$. The proton $\left(\mathrm{H}_{\mathrm{b}}\right)$ on the benzene core also slightly shifts from 7.57 to $7.50 \mathrm{ppm}$, owing to the rotation of $\mathrm{H}_{\mathrm{b}}$ away from DFIC. Nevertheless, aliphatic protons $\left(\mathrm{H}_{\mathrm{c}}\right.$ and $\left.\mathrm{H}_{\mathrm{e}}\right)$ have no obvious changes. Similar results are also observed from the temperature-dependent ${ }^{1} \mathrm{H}-\mathrm{NMR}$ of PTICH and PTICO (Supplementary Figs. 6-8).

From the ${ }^{13} \mathrm{C}-{ }^{1} \mathrm{H}$ correlation spectra of PTIC in $\mathrm{CDCl}_{3}$, the heteronuclear multiple-bond correlation (HMBC) exhibits a signal at $(7.71,154)$ of $\mathrm{H}_{\mathrm{a}}$ and $\mathrm{C}_{3}$ correlation (Fig. 2c, Supplementary Fig. 9), suggesting the existence of a preferential hydrogen-bond-based conformation. Still, the coexistence of S-O geometry in solution should not exclude due to the low rotation energy barrier of PT rotamer. To sum up, PTIC analogously adapts $\mathrm{O}-\mathrm{H}$ geometry as a preferential conformation, which may be still thermodynamically accessible to $\mathrm{O}-\mathrm{S}$ geometry in solution.

SSNMR of PTIC was further conducted to probe the molecular stacking behaviors in solids (Supplementary Figs. 10-14 and Supplementary Table 2). The results suggest "end-to-end" packing of PTIC in solid (Fig. 1b), which is similar, but in closer stacking distance to those reported FREAs (Supplementary Fig. 15) ${ }^{36,37}$. This observation is in agreement with GIWAXS measurements (which will be discussed later). From these theoretical and experimental results, we reveal that the NFRAs that have $\mathrm{O}-\mathrm{H}$ geometry as a preferential conformation, which may be rotatable in solution (due to the low-energy barrier of center single $\mathrm{C}-\mathrm{C}$ bond), can be restrained into a relatively rigid and planar structure with dense "end-to-end" stacking in solid. From the material processing point of view, this conformational rearrangement from solution to solid would endow NFRA acceptors not only good solution processability, but also excellent film optoelectronic properties.

Optoelectronic properties. The backbone conformation greatly influences optoelectronic properties of a molecule. NFRAs (PTICH, PTIC, and PTICO) have features of rotatable conformation in solution, while being restrained into a planar conformation upon stacking together in the film. The ultraviolet-visible (UV-vis) absorption spectra of three NFRAs and fused ID4F in solution and thin films are shown in Fig. 2e. In solution, PTICH, PTIC, and PTICO exhibit broad absorption peaks without apparent aggregation shoulders, with maximum absorption $\left(\lambda_{\mathrm{abs}}\right.$ sol) located at 637, 648, and $647 \mathrm{~nm}$ on the molar extinction coefficients $\left(\varepsilon_{\mathrm{abs}}{ }^{\mathrm{sol}}\right)$ of $0.92 \times 10^{5} \mathrm{M}^{-1} \mathrm{~cm}^{-1}$ (PTICH), $1.12 \times 10^{5} \mathrm{M}^{-1} \mathrm{~cm}^{-1}$ (PTIC), and $1.18 \times 10^{5} \mathrm{M}^{-1} \mathrm{~cm}^{-1}$ (PTICO), respectively. These extinction coefficients are 
a



b

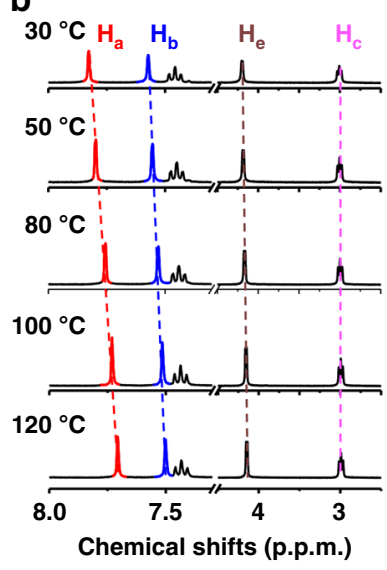

C

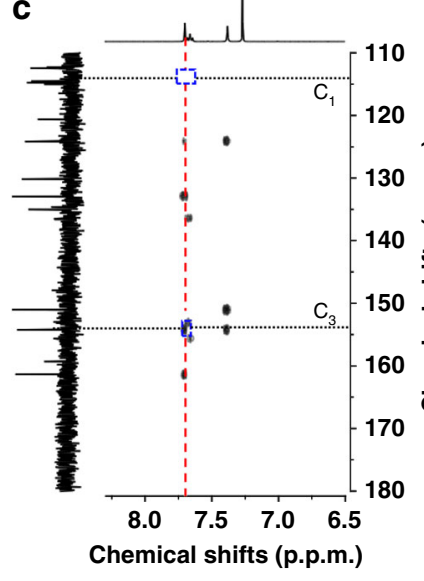

d

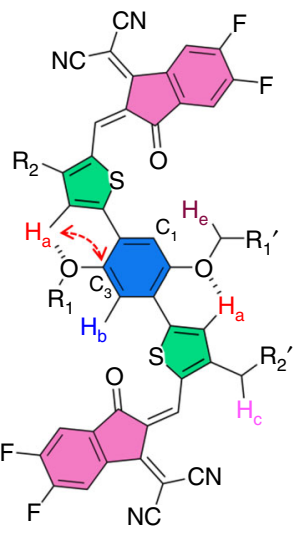

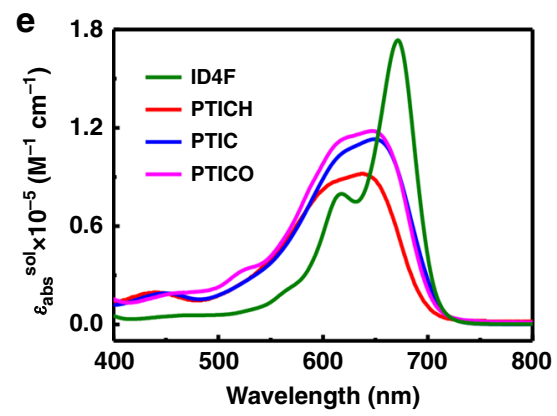
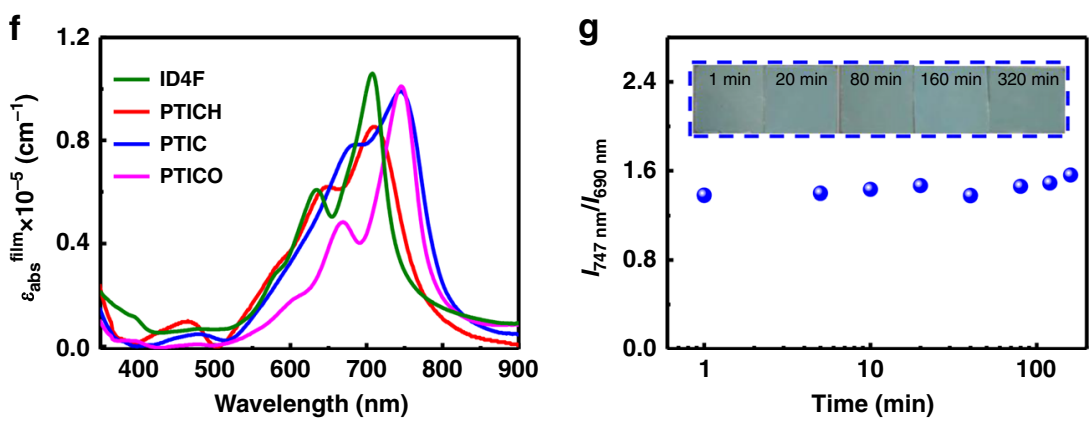

Fig. 2 Molecular conformation and optical properties. a Possible rotamers and their energy-torsion angle (E- $\theta$ ) curves: black curve for PT rotamer (left structure for $\mathrm{O}-\mathrm{H}$ geometry and $\mathrm{O}-\mathrm{S}$ geometry at the right); TICH rotamer (red), TIC rotamer (blue), and TICO rotamer (pink). b Temperature-dependent NMR of PTIC in $d_{4}-C_{6} D_{4} C l_{2}$. c The heteronuclear multiple-bond correlation (HMBC) of PTIC in $C C D C l_{3}$. d The preferential conformation of PTIC. e The solution and $\mathbf{f}$ film UV-vis absorption of ID4F and NFRAs. $\mathbf{g}$ The UV-vis absorption of $I_{747 \mathrm{~nm}} / I_{690 \mathrm{~nm}}$ ratio for PTIC film annealed at $100{ }^{\circ} \mathrm{C}$ for $320 \mathrm{~min}$, with an inset of optical images

\section{Table 1 Optical properties of ID4F and NFRAs}

\begin{tabular}{|c|c|c|c|c|c|c|c|c|}
\hline & $\lambda_{\mathrm{abs}} \mathrm{sol}(\mathrm{nm})$ & $\lambda_{\text {abs }}{ }^{\text {film }}(\mathrm{nm})$ & $\lambda_{\text {onset }^{\text {film }}}(\mathrm{nm})$ & $E_{\mathrm{g}}{ }^{\text {opt }}(\mathrm{eV})$ & $\lambda_{\mathrm{em}}{ }^{\mathrm{sol}}(\mathrm{nm})$ & $\lambda_{\mathrm{em}}^{\text {film }}(\mathrm{nm})$ & \multicolumn{2}{|c|}{ SS (nm) } \\
\hline ID4F & 671 & 707 & 752 & 1.64 & 725 & 758 & 54 & 51 \\
\hline PTIC & 650 & 747 & 810 & 1.53 & 725 & 795 & 75 & 48 \\
\hline PTICO & 647 & 746 & 794 & 1.56 & 718 & 795 & 71 & 49 \\
\hline
\end{tabular}

apparently lower than those of ID4F ( $\varepsilon_{\mathrm{abs}}$ sol of $1.75 \times 10^{5} \mathrm{M}^{-1} \mathrm{~cm}^{-1}$ at $671 \mathrm{~nm}$ ) in solution (Table 1 and Fig. 2e). It is because that fused ID4F has a rigid and planar structure in solution, ensuring stronger intramolecular charge transfer (ICT) effect and aggregation than those of non-planar NFRAs in solution.

Interestingly, the maximum absorption peaks of films $\left(\lambda_{\text {abs }}\right.$ film $)$ are observed at 709,747 , and $746 \mathrm{~nm}$ with strong aggregation shoulders for PTICH, PTIC, and PTICO, respectively (Fig. 2f). The $\lambda_{\text {abs }}$ film of NFRAs displays a large redshift over $70 \mathrm{~nm}$ from solution to the film, whereas ID4F has only a $36-\mathrm{nm}$ redshift. The extinction coefficients of films $\left(\varepsilon_{\mathrm{abs}}\right.$ film) of two NRFAs (PTIC, $0.99 \times 10^{5} \mathrm{~cm}^{-1}$ and PTICO, $1.01 \times 10^{5} \mathrm{~cm}^{-1}$ ) are almost in the same level of ID4F $\left(1.06 \times 10^{5} \mathrm{~cm}^{-1}\right)$. These indicate that NFRAs in films not only form dense J-aggregation, but also adapt planar conformation with enhanced ICTs, resulting in more bathochromic and hyperchromic shifts than ID4F. Besides, the PTIC shows excellent conformational and morphological stabilities in the film, as indicated from temperature-dependent absorption measurements (Fig. 2g).
When annealed from 25 to $200^{\circ} \mathrm{C}$, or at $100^{\circ} \mathrm{C}$ up to $360 \mathrm{~min}$, both the intensity ratios $\left(I_{747 \mathrm{~nm}} / I_{690 \mathrm{~nm}}\right)$ and absorption edge remain the same with no apparent changes (Fig. 2g, Supplementary Figs. 16 and 17). These results coincide with the differential scanning calorimetry (DSC) of acceptors without apparent thermal transitions below $200^{\circ} \mathrm{C}$ (Supplementary Fig. 18). On the contrary, in chlorobenzene (CB) solution, the $\lambda_{\mathrm{abs}}$ sol of PTIC is blue-shifted from 650 to $639 \mathrm{~nm}$ upon increasing temperature from room temperature to $70^{\circ} \mathrm{C}$, which may be attributed to flexible and rotational conformation in the solution (Supplementary Fig. 19).

The photoluminescence (PL) spectra of ID4F and NFRAs were also measured in both solution and film (Supplementary Fig. 20 and Table 1). The emission of PTIC shows the dependence of solvent polarity, attributing the ICT effect of NFRAs upon excitation (Supplementary Fig. 21). The Stokes shift (SS) can be calculated from the difference between maximum absorption and emission, which links to the reorganization energy of a molecule between ground and excited-state transition. In solution, the SS 
a

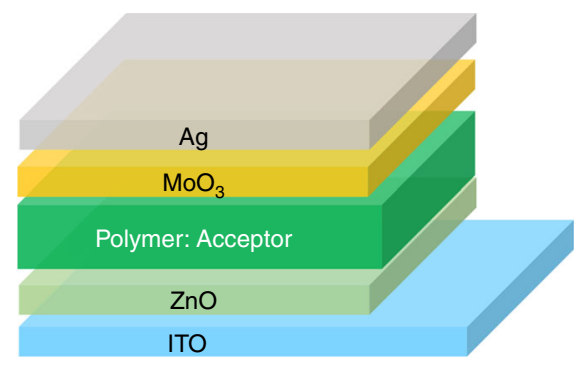

C

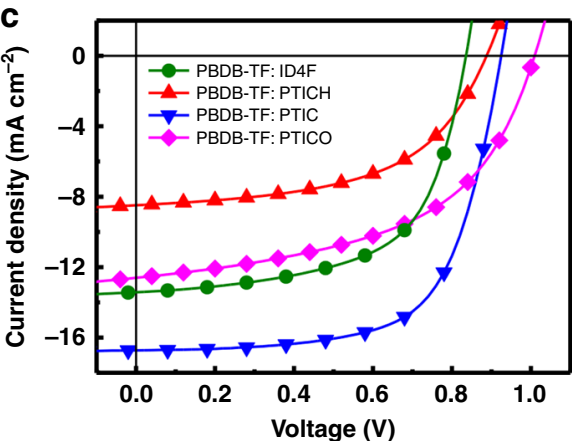

e

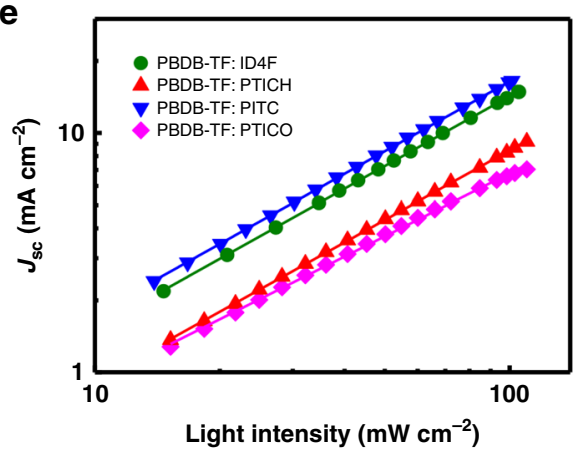

b

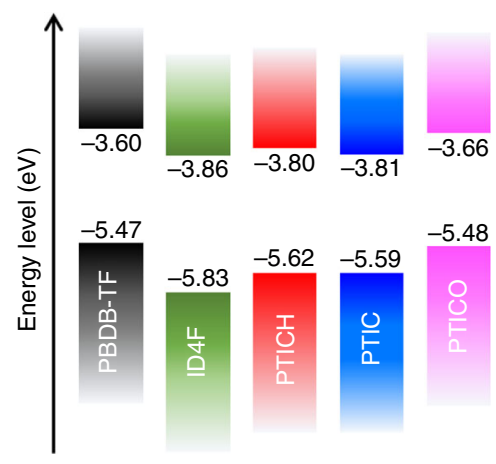

d

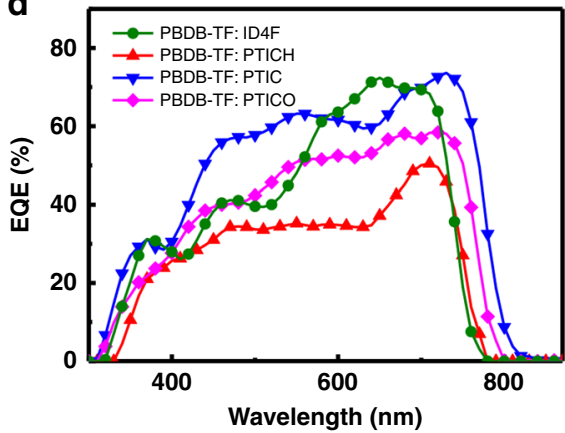

f



Fig. 3 Energy levels and photovoltaic performance. a Inverted OSC device architecture. b Energy levels for different active components. c The J-V characteristics and d EQE spectra of OSCs under AM $1.5 \mathrm{G}$ illumination $\left(100 \mathrm{~mW} \mathrm{~cm}^{-2}\right)$. e $J_{\mathrm{SC}}$ versus light intensity of the OSCs. $\mathbf{f}$ Stabilities of encapsulated devices under continuous illumination of a metal halide lamp without UV filtration. The error bars represent the standard deviation from four devices

values of PTICH, PTIC, and PTICO are 85,75 , and $71 \mathrm{~nm}$, which are all apparently larger than $54 \mathrm{~nm}$ of ID4F, indicating that the nonradiative transition of NFRAs consumes more energies than that of FREA ID4F. Interestingly, in the film, the SS values of NFRAs are largely reduced (48 nm for PTIC and $49 \mathrm{~nm}$ of PTICO), suggesting that NFRAs in the film have less (at least similar) nonradiative energy losses than those of ID4F $(51 \mathrm{~nm})$, which are consistent with the calculated reorganization energy values (Supplementary Fig. 22). This is in agreement with the quantum efficiencies of non-fused acceptors in the solid state, which are apparently higher than those in solution; attributing to molecular packing in solid helps rigidifying the molecular structure and suppressing nonradiative decay, over those of flexible structures in solution.

The energy levels of these acceptors were estimated from cyclic voltammetry $(\mathrm{CV})$ and ultraviolet photoelectron spectrometer (UPS) (Fig. 3b and Supplementary Table 3). NFRAs possess relatively upshifted energy levels over those of ID4F. They energetically match with the PBDB-TF polymer donor, except for PTICO, showing a small highest-occupied molecular orbital (HOMO) and the lowest-unoccupied molecular orbital offsets
(0.01 and $0.06 \mathrm{~V}$, respectively) with PBDB-TF (Supplementary Fig. 23).

Photovoltaic properties. To investigate the influence of NFRA structural factors to their photovoltaic performance, we have fabricated inverted OSCs with an architecture of indium tin oxide (ITO) $/ \mathrm{ZnO} /$ active layer $/ \mathrm{MoO}_{3} / \mathrm{Ag}$ (Fig. 3a), wherein the active layer employs the PBDB-TF polymer donor and each acceptor (Fig. 3b), respectively. Note that all devices are fabricated from single-solvent processing without an additive, which may be feasible and easy for scale-up fabrication. All NFRA-based blends are cast with $\mathrm{CB}$, and the ID4F blend is fabricated with $\mathrm{CHCl}_{3}$ due to its solubility preference. Their current density-voltage ( $J$ $V$ ) characteristics are shown in Fig. 3c, and device performances are summarized in Table 2. The ID4F-based device exhibited the PCE of $6.88 \%$, with the $J_{\mathrm{SC}}$ of $13.42 \mathrm{~mA} \mathrm{~cm}^{-2}, V_{\mathrm{OC}}$ of $0.84 \mathrm{~V}$, and FF of 0.61 . NFRA-based devices generally exhibit higher $V_{\mathrm{OC}}$ values $(0.92 \mathrm{~V}$ for PTICH, $0.93 \mathrm{~V}$ for PTIC, and $1.01 \mathrm{~V}$ for PTICO) with mitigated voltage losses $(0.68 \mathrm{~V}$ for PTICH, $0.58 \mathrm{~V}$ for PTIC, and $0.55 \mathrm{~V}$ for PTICO), comparing with those of fused ID4F (voltage losses of $0.80 \mathrm{~V}$ ). 
Table 2 The device parameters of OSCs with different acceptors under AM $1.5 \mathrm{G}$ illumination $\left(100 \mathrm{~mW} \mathrm{~cm}{ }^{-2}\right)$. The resistances were extracted from the $J-V$ curves under light illumination



Encouragingly, PTIC-based device shows the decent performance with PCE of $10.27 \%$, FF of 0.66 , and $J_{\mathrm{SC}}$ of $16.73 \mathrm{~mA} \mathrm{~cm}^{-2}$, and optimal series resistance $\left(R_{\mathrm{s}}\right)$, as well as shunt resistance $\left(R_{\mathrm{sh}}\right)$, despite that PTICH-based and PTICO-based devices possess relatively low $J_{\mathrm{SC}}$ and FF values. When a nonhalogenated solvent, such as $o$-xylene is employed, the PBDBTF: PTIC-based device can still reach 9.53\% PCE (Supplementary Fig. 24). The external quantum efficiency (EQE) spectra of the PBDB-TF: PTIC blend display a broadened photoresponse from 310 to $820 \mathrm{~nm}$ (Fig. $3 \mathrm{~d}$ ). The calculated $J_{\mathrm{SC}}$ values from the integration of EQE curves are $15.93 \mathrm{~mA} \mathrm{~cm}^{-2}$ (PBDB-TF: PTIC), $12.80 \mathrm{~mA} \mathrm{~cm}^{-2}$ (PBDB-TF: ID4F), $8.19 \mathrm{~mA} \mathrm{~cm}^{-2}$ (PBDB-TF: PTICH), and $12.18 \mathrm{~mA} \mathrm{~cm}^{-2}$ (PBDB-TF: PTICO), respectively, consistent with those from $J-V$ measurements.

It is interesting to note that the overall EQE and photocurrent of the PTIC-based device are superior to other devices in these studies. To understand the possible reasons, electron mobilities of blends are measured by the space charge-limited current (SCLC) method, revealing similar levels of $3.4 \times 10^{-5} \mathrm{~cm}^{2} \mathrm{~V}^{-1} \mathrm{~S}^{-1}$ (ID4F), $1.1 \times 10^{-4} \mathrm{~cm}^{2} \mathrm{~V}^{-1} \mathrm{~S}^{-1}$ (PTICH), $4.3 \times 10^{-5} \mathrm{~cm}^{2} \mathrm{~V}^{-1} \mathrm{~S}^{-1}$ (PTIC), and $1.7 \times 10^{-5} \mathrm{~cm}^{2} \mathrm{~V}^{-1} \mathrm{~S}^{-1}$ (PTICO), respectively. The corresponding hole mobilities are $2.5 \times 10^{-4} \mathrm{~cm}^{2} \mathrm{~V}^{-1} \mathrm{~S}^{-1}$ (ID4F), $2.3 \times 10^{-4} \mathrm{~cm}^{2} \mathrm{~V}^{-1} \mathrm{~S}^{-1} \quad$ (PTICH), $\quad 1.2 \times 10^{-4} \mathrm{~cm}^{2} \mathrm{~V}^{-1} \mathrm{~S}^{-1}$ (PTIC), and $1.7 \times 10^{-4} \mathrm{~cm}^{2} \mathrm{~V}^{-1} \mathrm{~S}^{-1}$ (PTICO). The ratios of charge carrier mobilities (electron mobility/hole mobility) were $0.13,0.47,0.37$, and 0.10 (Supplementary Fig. 25). The charge recombination of devices was further investigated via tracking the light intensity $\left(P_{\text {light }}\right)$-dependent $J-V$ characteristics ${ }^{26}$. As shown in Fig. $3 \mathrm{e}$, the slopes for $J_{\mathrm{SC}}-P_{\text {light }}$ were $0.97,0.96,0.98$, and 0.89 for ID4F-based, PTICH-based, PTIC-based, and PTICO-based devices, suggesting that all devices have good dissociation probabilities at short-circuit condition with slopes close to 1 , except for PBDB-TF: PTICO-based device (with a smaller slope of 0.89 ). It can be ascribed to the small energetic offsets between PTICO and polymer, perhaps hindering the exciton disassociation; thus there is a monomolecular recombination of the related OSCs, resulting in the moderate $J_{\mathrm{SC}}\left(12.60 \mathrm{~mA} \mathrm{~cm}^{-2}\right)$ and PCE (6.62\%). The dependence of $V_{\mathrm{OC}}$ on the $P_{\text {light }}$ reveals that the slopes of devices, are close to $1 k_{T} q^{-1}$ (Supplementary Fig. 26), indicating that open-circuit conduction devices have mainly a bimolecular recombination without trap-assisted Shockley-Read-Hall (SRH) recombination ${ }^{38}$.

In light of the decent performance obtained from simple PTIC NFRA, we studied the stabilities of devices (PBDB-TF: PTIC and PBDB-TF: ID4F) under continuous 1-sun-equivalent illumination. Note that a metal halide lamp without UV filtration is employed as a light source and its light density is monitored with a silicon cell. Surprisingly, PBDB-TF: PTIC-based device allows maintaining about $70 \%$ of its initial PCE value for $50-\mathrm{h}$ illumination, despite the harsh light source (containing a portion of high-energy UV photons) employed in studies. This is much better than that of PBDB-TF: ID4F-based devices (the remaining $25 \%$ of its initial value under the same condition). Four devices are used for standard deviation (Fig. 3f). To understand the intrinsic photostability of acceptors themselves, we have further compared the neat PTIC and ID4F films under constant 1-sunequivalent illumination, revealing that PTIC is more stable than that of ID4F. As shown in Supplementary Fig. 27, neat PTIC film remained as green color and there was a relatively steady absorption at $747 \mathrm{~nm}$, for $32-\mathrm{h}$ constant illumination. However, the fused ID4F and the original green film were faded to nearly transparent after 16-h illumination, indicating the breaking of a conjugated system. The thermal stress stability of PTIC-based and ID4F-based OSCs was investigated, and remains insensitive to thermal treatments at $100{ }^{\circ} \mathrm{C}$ for $32 \mathrm{~h}$ (Supplementary Fig. 28). The stability difference may stem from the structural factors of NFRA without extension-fused rings and tetrahedron $s p^{3}$ bridge carbon of FREA, as well as the capability of dense stacking in films, which helps gaining additional photostabilities of NFRAbased OSCs.

Blend morphology. The top-surface morphologies of NFRA blend films were measured by atomic force microscopy (AFM) (Supplementary Fig. 29). It reveals that PBDB-TF: PTICH film shows high surface roughness $\left(R_{\mathrm{q}}\right)$ of $21.6 \mathrm{~nm}$, indicating the strong aggregation of such blend and non-ideal morphology. For PTIC-based and PTICO-based blends, the film surfaces are relatively smooth with an $R_{\mathrm{q}}$ of $5.83 \mathrm{~nm}$ for PTIC and $7.73 \mathrm{~nm}$ for PTICO.

GIWAXS measurements were performed to study the molecular packing and crystallinity of the pure and blend films ${ }^{25,37}$. The intensity profiles in the out-of-plane and in-plane direction and 2D scattering patterns are shown in Fig. 4 and Supplementary Fig. 30, respectively. The peak positions and $d$-spacing are summarized in Supplementary Table 4. Pure ID4F film exhibits face-on orientation indicated by the $\pi-\pi$ peak located at $q_{\mathrm{z}}=1.66$ $\AA^{-1}(d=3.79 \AA)$, similar to most FREAs. The additional peak at $q_{\mathrm{z}}=0.825 \AA^{-1}$ corresponds to a layer spacing twice of the $\pi-\pi$ stacking distance, which is likely to originate from the spacing between the fused-ring core groups. Interestingly, all three NFRAs in neat films exhibit strong molecular stacking and high crystallinity, signified by sharp $\pi-\pi$ peaks along the $q_{\mathrm{r}}$ axis and up to three orders of lamellar peaks along the $q_{\mathrm{z}}$ axis. The $\pi-\pi$ stacking distances of PTICH, PTIC, and PTICO can be extracted as $3.63 \AA$ for PTICH, $3.59 \AA$ for PTIC, and $3.45 \AA$ for PTICO. Unlike a fused ladder structure, ID4F possesses two upward and downward pointing chains onto a tetrahedron $s p^{3}$ bridge carbon, NFRAs with alkoxy chains ensure shorter $\pi-\pi$ stacking distances. This feature is also beneficial to reduce the Stoke shifts of NFRAs in films.

The scattering profiles of blend films reveal that NFRA-based blends exhibit stronger lamellar peaks than the ID4F blend does, indicating higher crystallinity and more ordered stacking for NFRA blends. Upon blending, PBDB-TF:ID4F has a slightly increased acceptor lamellar distance $(15.0 \AA)$. The peak at 



Fig. 4 Morphologies of neat and blend films. The GIWAXS intensity profiles along the out-of-plane (a) and in-plane (b) directions
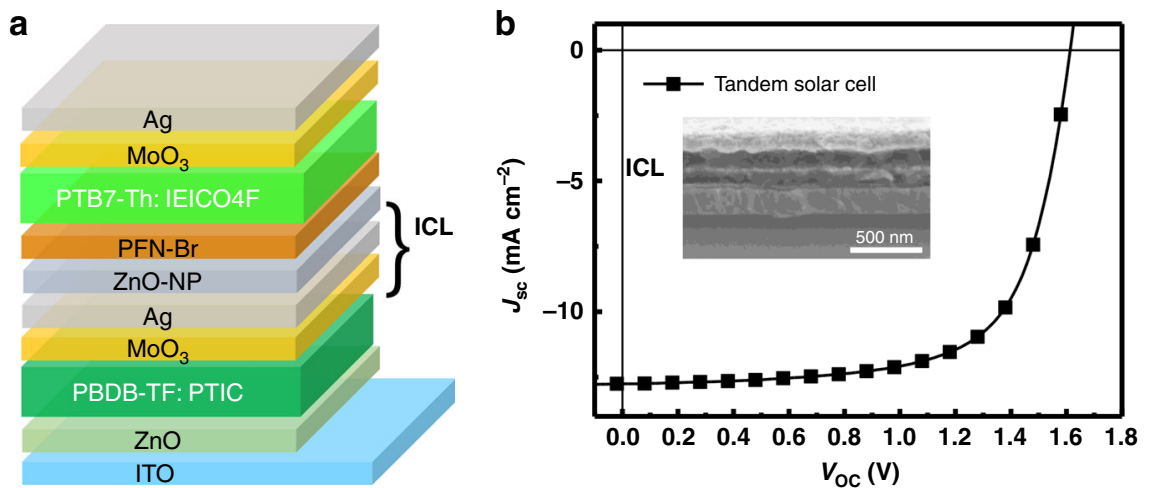

Fig. 5 Efficient tandem solar cell. a Monolithic tandem architecture and $\mathbf{b} \mathrm{J}-\mathrm{V}$ curves of the tandem device under the illumination of $\mathrm{AM} 1.5 \mathrm{G}\left(100 \mathrm{~mW} \mathrm{~cm}^{-2}\right.$ ). The inset is the cross-sectional SEM image for tandem OSCs

$q=0.305 \AA^{-1}$ corresponds to the lamellar stacking of the donor. NFRAs in blends still exhibit similar lamellar and $\pi-\pi$ stacking distances as in the neat films. It is evident that by removal of the covalent chemical bonding in fused-ring acceptors, NFRAs are given with a higher degree of freedom in solution, ultimately favoring the formation of higher crystallinity and tighter $\pi-\pi$ stacking, compared with the fused-ring counterpart.

As the PBDB-TF: PTIC BHJ showed efficient performance and a relatively high $V_{\mathrm{OC}}$ in single-junction OSCs, we further employed them into monolithic tandem OSCs with the architecture of ITO/ZnO/PBDB-TF: PTIC/ $\mathrm{MoO}_{3} / \mathrm{Ag} / \mathrm{NP}-\mathrm{ZnO} /$ PFN-Br/PTB7-Th: IEICO-4F/MoO $/$ /Ag (Fig. 5 and Supplementary Fig. 31). The interconnecting layer (ICL) is modified according to our previous approach ${ }^{27}$, wherein $0.5 \mathrm{~nm}$ of extra thin silver layer is employed for good charge recombination and light transmission. The tandem cells exhibited the $J_{\mathrm{SC}}$ of $12.76 \mathrm{~mA} \mathrm{~cm}^{-2}, V_{\mathrm{OC}}$ of $1.61 \mathrm{~V}$, and $\mathrm{FF}$ of 0.68 , resulting in the best PCE of $13.97 \%$ (PCE of $13.49 \%$ measured with aperture). It is highly encouraging that an exceptionally simple acceptor made from two-step synthesis allows leading to efficient and stable OSCs, which should be largely beneficial for their practical applications.

\section{Discussion}

In this work, simple and effective electron acceptors with a fully unfused backbone have been developed through a two-step synthesis from single aromatic units. Through the combined experimental and theoretical investigations, we reveal that those NFRAs have structural features of rotatable conformation in solution, while being restrained into a relatively rigid and planar conformation upon stacking in the film, with the assistance of intramolecular non-covalent interaction. As a result, NFRAs exhibit not only feasible solution processability, but also excellent optoelectronic and stacking properties in films. These are beneficial factors to fabricate efficient OSCs through simple solution processing. Note that non-fused PTIC shows efficient performance of $10.27 \%$ PCE in single-junction OSCs and $13.97 \%$ PCE in tandem OSCs with a low synthetic complexity index. In addition, NFRA-based OSCs exhibit promising stabilities under continuous illumination. Overall, our studies reveal valuable insights on the structural design of simple and effective molecules with great practical values.

\section{Methods}

General synthesis of NFRAs. The non-fused core, 5,5'-(2,5-bis((2-hexyldecyl) oxy)-1,4-phenylene)bis(3-substituent-2-thenaldehyde) (i.e., PTH-CHO or PT$\mathrm{CHO}$ or PTO-CHO) $(0.20 \mathrm{mmol})$, DFIC $(0.50 \mathrm{mmol})$, and a few drops of pyridine were dissolved in chloroform $\left(\mathrm{CHCl}_{3}\right)$. The reaction mixture was refluxed overnight, and then concentrated in vacuo. The solid crude products were obtained through methanol precipitation, and washed with methanol and acetone, respectively. The final crystalline solid was obtained through the recrystallization from $\mathrm{CHCl}_{3}$ and methanol, and washed with a mixture of $\mathrm{CHCl}_{3} /$ methanol $(v / v=1 / 1)$ to obtain pure product. The detailed synthesis is described in Supplementary 
Methods, and the final products were characterized by ${ }^{1} \mathrm{H}-\mathrm{NMR},{ }^{13} \mathrm{C}-\mathrm{NMR}$, and mass spectra (Supplementary Figs. 35-44).

Single-junction cell fabrication. Polymer solar cells were fabricated on glass substrates commercially pre-coated with a layer of ITO with the inverted structure of ITO/ZnO/PBDB-TF: Acceptor $/ \mathrm{MoO}_{3} / \mathrm{Ag}$. Prior to fabrication, the substrates were cleaned using detergent, deionized water, acetone, and isopropanol consecutively for every $15 \mathrm{~min}$, and then treated in an ultraviolet ozone generator for $15 \mathrm{~min}$. A thin layer of sol-gel $\mathrm{ZnO}$ was spin-coated onto clean ITO-coated glass substrates at $3500 \mathrm{rpm}$ for $60 \mathrm{~s}$ and then annealed at $170^{\circ} \mathrm{C}$ for $20 \mathrm{~min}$; then the substrates were transferred to a glovebox. The PBDB-TF: ID4F in $\mathrm{CHCl}_{3}$ (16 $\left.\mathrm{mg} \mathrm{mL}^{-1}, 1: 1.2 \mathrm{wt} \%\right)$ and PBDB-TF: NFRAs in CB $\left(20 \mathrm{mg} \mathrm{mL}^{-1}, 1: 1.2 \mathrm{wt} \%\right)$ were then spin-coated on the $\mathrm{ZnO}$ layers. Then the $\mathrm{BHJ}$ layers were annealed at $100{ }^{\circ} \mathrm{C}$ under nitrogen atmosphere. The, the $\mathrm{MoO}_{3}$ layer $(4 \mathrm{~nm})$ was deposited on the active layers. Finally, silver $(100 \mathrm{~nm})$ was thermally evaporated through shadow masks to complete the device with an active area of $6 \mathrm{~mm}^{2}$.

Tandem cell fabrication. The active layer for the front cell was first spin-coated atop of $\mathrm{ZnO} / \mathrm{ITO}$ substrates from PBDB-TF: PTIC in CB $\left(20 \mathrm{mg} \mathrm{mL}^{-1}, 1: 1.2 \mathrm{wt} \%\right)$ at $2000 \mathrm{rpm}$ for $60 \mathrm{~s}$, and annealed at $120^{\circ} \mathrm{C}$ for $10 \mathrm{~min}$. Then, $\mathrm{MoO}_{3}(4 \mathrm{~nm})$ and ultrathin $\mathrm{Ag}(0.5 \mathrm{~nm})$ were deposited via thermal evaporation. The deposition of the ultrathin Ag layer was defined by a mask with good alignment to the patterned ITO strip. Atop of an ultrathin Ag layer, $\mathrm{ZnO}-\mathrm{NP}$ and PFN-Br in methanol were subsequently spin-coated to complete ICL fabrication. Afterward, the active layer for the rear cell was spin-coated from PTB7-Th: IEICO-4F in CB $(5 \% \mathrm{CN})$ (30 $\mathrm{mg} \mathrm{mL}^{-1}, 1: 1.5 \mathrm{wt} \%$ ) at $3000 \mathrm{rpm}$ for $60 \mathrm{~s}$. The tandem device was completed by evaporating $4 \mathrm{~nm}$ of $\mathrm{MoO}_{3}$ and $100 \mathrm{~nm}$ of Ag. The active area of tandem cells is $6 \mathrm{~mm}^{2}$ (without aperture) and $3.75 \mathrm{~mm}^{2}$ (with aperture).

J-V measurements. The current density-voltage $(J-V)$ curves of OSCs were measured with Keithley 2400, under AM $1.5 \mathrm{G}$ illumination at $100 \mathrm{~mW} \mathrm{~cm} \mathrm{~cm}^{-2}$ irradiation using a Enli SS-F5-3A solar simulator, and the light intensity was calibrated with a standard Si solar cell with a KG5 filter (made by Enli Technology Co., Ltd., Taiwan, and a calibrated report can be traced to NREL). The EQE spectrum was measured using a QE-R Solar Cell Spectral Response Measurement System (Enli Technology Co., Ltd., Taiwan). The EQE spectrum for the rear and front cells of tandem OSC was measured under the light bias obtained from 550-nm low-pass and 850-nm high-pass optical filters (upon exciting the front and rear cells), respectively. The mismatch factor (MM) values of the front cell and back cell are calculated to be 1.006 and 1.076, respectively, from the following equation:

$$
\mathrm{MM}=\frac{\int E_{\text {ref }}(\lambda) S_{\text {ref }}(\lambda) \mathrm{d} \lambda \cdot \int E_{\text {meas }}(\lambda) S_{\text {sample }}(\lambda) \mathrm{d} \lambda}{\int E_{\text {meas }}(\lambda) S_{\text {ref }}(\lambda) \mathrm{d} \lambda \cdot \int E_{\text {ref }}(\lambda) S_{\text {sample }}(\lambda) \mathrm{d} \lambda}
$$

$E_{\text {ref }}(\lambda)$ is reference spectral irradiance of AM $1.5 \mathrm{G}$ spectrum, $E_{\text {meas }}(\lambda)$ is the spectrum of the testing solar simulator; $S_{\text {ref }}(\lambda)$ and $S_{\text {sample }}(\lambda)$ are the spectrum response of a standard cell used to calibrate the simulator light intensity, and the spectral response of the sample solar cell under test, respectively.

Device stability test. OSC devices are sealed with a cover glass by an UV-curing adhesive of epoxy resin LX803 (purchased from Solarmer Company). The stability of the encapsulated devices was measured in air under continuous 1 sun-equivalent illumination provided by a metal halide lamp (PHILIPS MSR 1200HR) without UV filtration (temperature: $45 \pm 5^{\circ} \mathrm{C}$ ). Light intensity was monitored by standard silicon solar cells.

\section{Data availability}

The data that support the findings of this study are available from the corresponding author upon reasonable request.

Received: 5 December 2018 Accepted: 20 April 2019

Published online: 14 May 2019

\section{References}

1. Hou, J., Inganas, O., Friend, R. H. \& Gao, F. Organic solar cells based on nonfullerene acceptors. Nat. Mater. 17, 119-128 (2018).

2. Yan, C. et al. Non-fullerene acceptors for organic solar cells. Nat. Rev. Mater. 3, 18003-18022 (2018)

3. Li, C.-Z., Yip, H.-L. \& Jen, A. K. Y. Functional fullerenes for organic photovoltaics. J. Mater. Chem. 22, 4161-4178 (2012).

4. Zhao, J. et al. Efficient organic solar cells processed from hydrocarbon solvents. Nat. Energy 1, 15027 (2016).

5. Huang, J. et al. Highly efficient organic solar cells consisting of double bulk heterojunction layers. Adv. Mater. 29, 1606729 (2017).
6. Deng, D. et al. Fluorination-enabled optimal morphology leads to over $11 \%$ efficiency for inverted small-molecule organic solar cells. Nat. Commun. 7, 13740-13749 (2016).

7. Cheng, P., Li, G., Zhan, X. \& Yang, Y. Next-generation organic photovoltaics based on non-fullerene acceptors. Nat. Photonics 12, 131-142 (2018).

8. Zhang, S., Qin, Y., Zhu, J. \& Hou, J. Over $14 \%$ efficiency in polymer solar cells enabled by a chlorinated polymer donor. Adv. Mater. 30, 1800868 (2018).

9. Fan, B. et al. Fine-tuning of the chemical structure of photoactive materials for highly efficient organic photovoltaics. Nat. Energy 3, 1051-1058 (2018).

10. Lin, Y. et al. An electron acceptor challenging fullerenes for efficient polymer solar cells. Adv. Mater. 27, 1170-1174 (2015)

11. Lin, Y. et al. High-performance fullerene-free polymer solar cells with $6.31 \%$ efficiency. Energy Environ. Sci. 8, 610-616 (2015).

12. Yao, H. et al. Design and synthesis of a low bandgap small molecule acceptor for efficient polymer solar cells. Adv. Mater. 28, 8283-8287 (2016).

13. Huang, C. et al. Highly efficient organic solar cells based on S,N-heteroacene non-fullerene acceptors. Chem. Mater. 30, 5429-5434 (2018).

14. Holliday, S. et al. High-efficiency and air-stable P3HT-based polymer solar cells with a new non-fullerene acceptor. Nat. Commun. 7, 11585-11596 (2016).

15. Lin, Y. et al. A facile planar fused-ring electron acceptor for as-cast polymer solar cells with 8.71\% efficiency. J. Am. Chem. Soc. 138, 2973-2976 (2016).

16. Wang, W. et al. Fused hexacyclic nonfullerene acceptor with strong nearinfrared absorption for semitransparent organic solar cells with $9.77 \%$ efficiency. Adv. Mater. 29, 1701308 (2017).

17. Liu, F. et al. Efficient semitransparent solar cells with high NIR responsiveness enabled by a small-bandgap electron acceptor. Adv. Mater. 29, 1606574 (2017).

18. Li, Y. et al. High efficiency near-infrared and semitransparent non-fullerene acceptor organic photovoltaic cells. J. Am. Chem. Soc. 139, 17114-17119 (2017).

19. Li, H., Lu, K. \& Wei, Z. Polymer/small molecule/fullerene based ternary solar cells. Adv. Energy Mater. 7, 1602540 (2017).

20. Yao, Z. et al. Dithienopicenocarbazole-based acceptors for efficient organic solar cells with optoelectronic response over $1000 \mathrm{~nm}$ and an extremely low energy loss. J. Am. Chem. Soc. 140, 2054-2057 (2018).

21. Xiao, Z., Jia, X. \& Ding, L. Ternary organic solar cells offer $14 \%$ power conversion efficiency. Sci. Bull. 62, 1562-1564 (2017).

22. Li, S., Liu, W., Li, C.-Z., Shi, M. \& Chen, H. Efficient organic solar cells with non-fullerene acceptors. Small 13, 1701120 (2017).

23. Meng, L. et al. Organic and solution-processed tandem solar cells with $17.3 \%$ efficiency. Science 361, 1094-1100 (2018).

24. Min, J. et al. Evaluation of electron donor materials for solution-processed organic solar cells via a novel figure of merit. Adv. Energy Mater. 7, 1700465 (2017).

25. Mai, J. et al. Understanding morphology compatibility for high-performance ternary organic solar cells. Chem. Mater. 28, 6186-6195 (2016).

26. Sun, C. et al. A low cost and high performance polymer donor material for polymer solar cells. Nat. Commun. 9, 743-753 (2018).

27. Chen, F.-X. et al. Near-infrared electron acceptors with fluorinated regioisomeric backbone for highly efficient polymer solar cells. Adv. Mater. 30 1803769 (2018).

28. $\mathrm{Li}, \mathrm{S}$. et al. Revealing the effects of molecular packing on the performances of polymer solar cells based on A-D-C-D-A type non-fullerene acceptors. J. Mater. Chem. A 6, 12132-12141 (2018).

29. Yi, Y.-Q.-Q. et al. Small molecule acceptors with a nonfused architecture for high-performance organic photovoltaics. Chem. Mater. 31, 904-911 (2019).

30. Zhang, Z. et al. A simple electron acceptor with unfused backbone for polymer solar cells. Acta Phys.-Chim. Sin. 35, 394-401 (2019).

31. Nguyen, T. L. et al. Semi-crystalline photovoltaic polymers with efficiency exceeding $9 \%$ in a $\sim 300 \mathrm{~nm}$ thick conventional single-cell device. Energy Environ. Sci. 7, 3040-3051 (2014).

32. Liu, Y. et al. Exploiting noncovalently conformational locking as a design strategy for high performance fused-ring electron acceptor used in polymer solar cells. J. Am. Chem. Soc. 139, 3356-3359 (2017).

33. Huang, H., Yang, L., Facchetti, A. \& Marks, T. J. Organic and polymeric semiconductors enhanced by noncovalent conformational locks. Chem. Rev. 117, 10291-10318 (2017).

34. Du, X. et al. Efficient polymer solar cells based on non-fullerene acceptors with potential device lifetime approaching 10 years. Joule 3, 215-226 (2018).

35. Sigalov, M. V., Doronina, E. P. \& Sidorkin, V. F. C(Ar)-H...O hydrogen bonds in substituted isobenzofuranone derivatives: geometric, topological, and NMR characterization. J. Phys. Chem. A 116, 7718-7725 (2012).

36. Shi, X. et al. Design of a highly crystalline low-band gap fused-ring electron acceptor for high-efficiency solar cells with low energy loss. Chem. Mater. 29, $8369-8376$ (2017). 
37. Mai, J. et al. Hidden structure ordering along backbone of fused-ring electron acceptors enhanced by ternary bulk heterojunction. Adv. Mater. 30, 1802888 (2018).

38. Cowan, S. R., Roy, A. \& Heeger, A. J. Recombination in polymer-fullerene bulk heterojunction solar cells. Phys. Rev. B 82, 245207 (2010).

\section{Acknowledgements}

This research was funded by National Natural Science Foundation of China (Nos. 21722404, 21674093, and 21734008), International Science and Technology Cooperation Program of China (ISTCP) (Grant no. 2016YFE0102900), Zhejiang Province Science and Technology Plan (no. 2018C01047), and supported by the Fundamental Research Funds for the Central Universities (2018XZZX002-16). C.-Z.L. thanks the support by Zhejiang Natural Science Fund for Distinguished Young Scholars (LR17E030001). X.K. thanks the support by Zhejiang Provincial Natural Science Foundation R19B050003, Zhejiang University K.P. Chao's High Technology Development Foundation, X.L. thanks Research Grant Council of Hong Kong (Theme-based Research Scheme nos. T23-407/13-N and 14314216). Z.-P.Y. thanks the support by the China Postdoctoral Science Foundation Funded Project (512300-X91803) and Postdoctoral Science Foundation Funded Project of Zhejiang Province (512300-X81701).

\section{Author contributions}

C.-Z.L. developed the concept and designed the experiments. C.-Z.L. and H.C. supervised the project. Z.-P.Y. designed and performed chemical synthesis and properties characterization of NFRAs. Z.-X.L. and F.-X.C. carried out the OSC fabrication and char acterization. R.Q. synthesized and characterized the fused ID4F. T.-K.L. and X.-H.L. performed GIWAXS measurements and analysis. J.-L.Y. and X.K. contribute to the SSNMR and analysis. Z.-P.Y., C.-Z.L., Z.-X.L., M.S., and H.C. analyzed the results and prepared the paper. All authors commented on the paper.

\section{Additional information}

Supplementary Information accompanies this paper at https://doi.org/10.1038/s41467019-10098-Z.

Competing interests: The authors declare no competing interests.

Reprints and permission information is available online at http://npg.nature.com/ reprintsandpermissions/

Journal peer review information: Nature Communications thanks Zhishan Bo, Ning Li and Andrew Wadsworth for their contribution to the peer review of this work.

Publisher's note: Springer Nature remains neutral with regard to jurisdictional claims in published maps and institutional affiliations.

\begin{abstract}
(c) ()
Open Access This article is licensed under a Creative Commons Attribution 4.0 International License, which permits use, sharing, adaptation, distribution and reproduction in any medium or format, as long as you give appropriate credit to the original author(s) and the source, provide a link to the Creative Commons license, and indicate if changes were made. The images or other third party material in this article are included in the article's Creative Commons license, unless indicated otherwise in a credit line to the material. If material is not included in the article's Creative Commons license and your intended use is not permitted by statutory regulation or exceeds the permitted use, you will need to obtain permission directly from the copyright holder. To view a copy of this license, visit http://creativecommons.org/ licenses/by/4.0/.
\end{abstract}

(C) The Author(s) 2019 